ciación directa entre la fluorosis $(P<0,05)$ y a) un mayor consumo de agua para la reconstitución de las fórmulas alimentarias a los 3, 6 y 9 meses de edad; b) un mayor consumo de agua a los 16 meses; y c) un mayor consumo combinado de jugos y otras bebidas a los 16 meses. El consumo de fluoruro con las bebidas fue también mayor en los niños con fluorosis que en los que no presentaban esta afección.

Los modelos de regresión logística multifactorial permitieron determinar que un mayor consumo de agua con las fórmulas reconstituidas se asociaba de manera directa con la aparición de fluorosis (a diferencia de los casos que no consumieron agua o que la consumieron en menor cantidad) a los 3 meses $(P<0,05)$, a los 6 meses $(P<0,001)$, a los 9 meses $(P<0,001)$ y a los 12 meses $(P<0,05)$ de edad. De manera similar, la cantidad de fluoruro consumido en el agua mostró una asociación directa con la presencia de fluorosis a los 16 meses de edad, $P<0,01)$.

También se observó una asociación directa con la presencia de fluorosis entre los 6 y los 16 meses de edad en los niños: a) que tuvieron un elevado consumo de fluoruro debido a la elevada concentración que tenía el agua empleada para reconstituir las fórmulas $(P<0,001)$; b) que consumieron cantidades mayores de fluoruro con el agua que bebían $(P<0,05)$; y c) que consumieron fluoruro, en cualquier cantidad, en los suplementos alimentarios $(P<0,001)$.

El consumo de fórmulas lácteas reconstituidas a partir de polvos mostró una asociación con la presencia de fluorosis, tanto en los niños que consumieron cantidades normales de fórmula preparada con agua con concentraciones elevadas de fluoruro, como en los que consumieron cantidades excesivas de fórmula preparada con agua con concentraciones menores de fluoruro.

El uso de dentífricos fluorados no pareció asociarse con una mayor frecuencia de fluorosis en los dientes primarios en las edades estudiadas.

Las concentraciones de fluoruro, tanto en la leche materna (de 0,005 a 0,010 partes por millón [ppm]) como en la leche de vaca (de 0,03 a 0,06 ppm), fueron bajas y en la cohorte estudiada, el consumo de leche de vaca mostró una asociación inversa con la presencia de fluorosis.

Estos resultados apoyan la hipótesis de que las bebidas consumidas durante el primer año de vida influyen en el riesgo de sufrir fluorosis de los dientes primarios. Estos hallazgos son importantes debido a que la fluorosis de los dientes primarios es un factor pronóstico en relación con la fluorosis de los dientes permanentes. La comprensión de los mecanismos que facilitan la fluorosis primaria y la identificación de los niños en riesgo de contraer esta afección pueden ayudar a mejorar las recomenda- ciones elaboradas para reducir el riesgo de fluorosis en los dientes permanentes.

Estos resultados indican que las altas concentraciones de fluoruro en el agua utilizada para reconstituir las fórmulas alimentarias pueden elevar el riesgo de fluorosis, por lo que se deberían idear intervenciones específicas para contrarrestar el problema. (Marshall TA, Levy SM, Warren JJ, Brofitt B, Eichenberger-Gilmore JM, Stumbo PJ. Associations between intakes of fluoride from beverages during infancy and dental fluorosis of primary teeth. J Am Coll Nutr. 2004;23:108-16.)

\section{El cáncer en niños en Centroamérica y el Caribe}

Con frecuencia, los pacientes pediátricos que tienen cáncer no reciben el tratamiento óptimo debido a la falta de recursos y a las deficiencias organizativas de los centros asistenciales. Para evaluar la magnitud de este problema se decidió llevar a cabo una evaluación epidemiológica del impacto de la atención sanitaria en la evolución final de estos pacientes. Este estudio se realizó en el marco de un proyecto internacional de colaboración dirigido a facilitar la transferencia de los protocolos diagnósticos y terapéuticos entre centros de excelencia de Italia y de Centroamérica y el Caribe mediante programas de fraternidad.

Entre los objetivos de este trabajo estaba el de documentar la magnitud y determinar las posibles causas de la brecha que se observa en las tasas de supervivencia a los 3 años del diagnóstico en niños con cáncer en países desarrollados y en países en desarrollo, aplicando las pautas terapéuticas actuales.

Se realizó una encuesta retrospectiva multicéntrica que abarcaba a todos los pacientes pediátricos diagnosticados y tratados de manera consecutiva en ocho hospitales de alcance nacional en siete países de Centroamérica y el Caribe: Costa Rica, Cuba, El Salvador, Guatemala, Honduras, Nicaragua y la República Dominicana. Estos centros formaban parte de una red de hospitales en el campo de la hematología y oncología en Centroaérica y el Caribe que se estableció en 1996 bajo los auspicios de la Escuela Internacional de Hematología-Oncología Pediátricas de Monza, Italia (MISPHO). El protocolo de investigación se discutió ampliamente entre todos los participantes, por lo que los datos que se recogieron y los criterios empleados para evaluarlos estaban predefinidos con toda claridad.

Se analizaron 2214 pacientes con trastornos hematológicos y oncológicos (70\%) y tumores sólidos (30\%) diagnosticados entre 1996 y 1999. Los datos demográficos (fecha de nacimiento y sexo) y clínicos (fecha y tipo de diagnóstico, fecha de la última consulta de seguimiento, estado vital, y notifi- 
cación y razones para abandonar el seguimiento) se tomaron de las historias clínicas. Los formularios llenados por los médicos se enviaron al centro coordinador en Monza para comprobar la coherencia de los datos. Las dudas surgidas se aclararon con los propios investigadores de manera individual. El seguimiento se extendió hasta el 30 de junio de 2000.

Se registraron 477 casos de abandono del seguimiento, $67 \%$ de ellos durante los tres primeros meses después del diagnóstico. Solo 40 casos $(8,4 \%)$ abandonaron el seguimiento al cabo de un año de haberse hecho el diagnóstico. El abandono del seguimiento puede considerarse un fracaso terapéutico, ya que lo más probable es que estos casos hayan muerto debido a la progresión de la enfermedad y a la falta de atención médica.

La supervivencia general a los tres años fue de $65,6 \%$ (error estándar $[\mathrm{ES}]=2,0$ ) cuando se excluyeron de los cálculos los casos de abandono del seguimiento $(16 \%)$, pero descendió a $54,2 \%$ (ES = $1,9)$ cuando los casos de abandono se sumaron a los fracasos terapéuticos.

El análisis detallado de los casos con leucemia linfoblástica aguda reveló una gran variabilidad entre los diferentes países que participaron en el estudio en cuanto a la supervivencia a los 3 años: $62,2 \%(\mathrm{ES}=5,3)$ en Cuba, $74,2 \%(\mathrm{ES}=3,3)$ en Costa Rica y $61,7 \%(E S=4,9)$ en Nicaragua, cifras todas muy superiores a las de los otros cuatro países participantes. Estos resultados pueden atribuirse a la baja proporción de casos que abandonaron el seguimiento en estos países $(0,9 \%, 2,7 \%$ y $7,5 \%$, respectivamente). En el caso de Cuba y Costa Rica, la alta retención de los casos durante el seguimiento podría deberse a la buena calidad de sus sistemas de salud, mientras que los pocos abandonos del seguimiento en Nicaragua posiblemente se debieron a los avances logrados recientemente por un programa nacional de hematología y oncología pediátrica. En países donde el número de casos que abandonaron el seguimiento en los primeros meses fue elevado se observaron las menores cifras de supervivencia a $\operatorname{los} 3$ años.

De estos resultados se pueden extraer dos conclusiones fundamentales: a) la curva general de supervivencia confirma y cuantifica la brecha que existe, en lo que respecta al derecho a la vida, entre los niños con cáncer que viven en países con menos recursos económicos y los que viven en países industrializados; b) las diferencias observadas en la supervivencia, incluso entre países de la misma región geográfica y cultural, hacen pensar que estos resultados no están tan vinculados con las condiciones macroeconómicas como con la existencia de una actitud de responsabilidad hacia la atención médica de los que la necesitan. Los casos de abandono temprano (durante el primer mes después del diagnóstico) constituyen la mayor proporción de casos con desenlaces desfavorables evitables.

Una larga tradición de atención continuada, respaldada por el concepto de que la salud es un derecho básico de las personas, genera perfiles de supervivencia similares en algunos países donde el tratamiento de los trastornos hematológicos y oncológicos, a pesar de su elevado costo, se considera esencial para la vida de una pequeña minoría compuesta de niños con cáncer. La promisoria situación de Nicaragua se debe en parte a la existencia de un proyecto de colaboración que garantiza la disponibilidad de medicamentos, pero más aun a la estrategia altamente participativa que se ha adoptado en ese país, en la que los padres toman parte en actividades de autoayuda que proveen las bases logísticas para poder cumplir con las diferentes etapas del tratamiento. (Valsecchi MG, Tognoni G, Bonilla M, Moreno N, Báez F, Pacheco C, et al. Clinical epidemiology of childhood cancer in Central America and Caribbean countries. Ann Oncol. 2004;15:680-5.)

\section{La relación del ambiente con la leptospirosis en Iquitos, Perú}

La leptospirosis es una zoonosis que se presenta de forma endémica o epidémica, tanto en áreas urbanas como rurales, en todas partes del mundo. La transmisión de Leptospira y la expresión clínica de la enfermedad varían en función del entorno ambiental y socioeconómico. La leptospirosis epidémica se acompaña de hemorragia pulmonar, insuficiencia renal e ictericia y es más frecuente en las áreas urbanas, donde el nivel de protección inmunitaria de los habitantes es más heterogéneo. Por el contrario, en las áreas rurales de los países en desarrollo se observa una mayor asociación entre la seropositividad a esta infección y la enfermedad subclínica.

El entorno ambiental de Iquitos, Perú, en la cuenca del Amazonas, presenta condiciones ideales para la transmisión de Leptospira, no solo debido al calor y a la humedad tropical predominantes, sino también a la elevada densidad poblacional y a la abundancia de mamíferos que pueden servir de vectores. Se ha observado que aproximadamente $30 \%$ de las personas de esta región que han padecido de fiebre aguda sin causa conocida presentan resultados serológicos que apuntan a la presencia de leptospirosis aguda.

El objetivo de este trabajo fue determinar la posible relación del ambiente con la exposición a Leptospira. Para ello se realizó un estudio seroepidemiológico transversal de base poblacional en tres contextos epidemiológicos diferentes del Perú: uno de alta prevalencia (Belén, un barrio marginal 